\title{
Proposta de uma cartilha educativa para pacientes acamados sobre medidas
}

\section{preventivas de lesão por pressão}

\author{
Proposal for an educational booklet for binded patients on preventive measures for pressure injury \\ Propuesta de folleto educativo para pacientes encamados sobre medidas preventivas
}

\author{
Anderson Nascimento da Conceição \\ ORCID: https://orcid.org/0000-0002-4695-9415 \\ Centro Universitário da Amazônia, Brasil \\ E-mail: adnascimento24@gmail.com \\ Italo Vinicius Regis Dos Santos \\ ORCID: https://orcid.org/0000-0002-2732-6778 \\ Centro Universitário da Amazônia, Brasil \\ E-mail: Italovinicius1204@gmail.com \\ Regiane de Paula Maciel \\ ORCID: https://orcid.org/0000-0002-4072-085X \\ Centro Universitário da Amazônia, Brasil \\ E-mail: regianestm@hotmail.com
}

\begin{abstract}
Resumo
Introdução: A prevalência dos casos de lesão por pressão é exorbitante, e a prevenção das LPPs continua sendo um grande desafio para os enfermeiros, e sua incidência é considerado um indicador de má qualidade da assistência. Objetivos: Contudo o objetivo do estudo foi elaboras uma cartilha educativa enfatizando as medidas preventivas de lesão por pressão em pacientes acamados. Metodologia: Trata-se de uma pesquisa qualitativa do tipo bibliográfica em que foi realizada a proposta de uma cartilha educativa para pacientes acamados. Entretanto o percurso metodológico utilizado abordará pesquisas bibliográficas como referencia á realização da mesma. Para atingir os objetivos propostos foi realizado uma busca no banco de dados, Google Acadêmico; BVS (Biblioteca Virtual em Saúde), SCIELO (Scientific Electronic Library Online), PubMed; LILACS (Literatura Latino-Americana e do Caribe em Ciências da Saúde). resultados e discussão: O material apresentado e desenvolvido para educação dos pacientes, foi apontado através de uma cartilha educativa. O material de educação em saúde deve ser redigido de forma simples, com menor nível de leitura e que permita transmitir informações precisas. As ilustrações devem ser atrativas com comunicação clara de sua finalidade. Além disso, as imagens devem atingir alto nível de atenção e interesse na leitura do material com alteração da população em diferentes níveis de escolaridade. Conclusão: O estimulo ao autocuidado com a prevenção minimiza os índices de morbimortalidade relacionada às infecções dos pacientes com LPP, diminui os gastos com saúde e melhora a qualidade de vida dos pacientes com LPP.
\end{abstract}

Palavras-chave: Lesão por pressão; Enfermagem; Educação em saúde.

\begin{abstract}
Introduction: The prevalence of pressure injury cases is exorbitant, and the prevention of PPLs remains a great challenge for nurses, and its incidence is considered an indicator of poor quality of care. Objectives: However, the aim of the study was to prepare an educational booklet emphasizing preventive measures for pressure injuries in bedridden patients. Methodology: This is a qualitative bibliographic research in which an educational booklet for bedridden patients was proposed. However, the methodological approach used will address bibliographic research as a reference for carrying out the same. To achieve the proposed objectives, a search was carried out in the database, Google Academic; VHL (Virtual Health Library), SCIELO (Scientific Electronic Library Online), PubMed; LILACS (Latin American and Caribbean Literature on Health Sciences). results and discussion: The material presented and developed for patient education was appointed through an educational booklet. The health education material must be written in a simple way, with a lower level of reading and allowing accurate information to be conveyed. Illustrations must be attractive with clear communication of their purpose. In addition, the images must reach a high level of attention and interest in reading the material, with changes in the population at different levels of education. Conclusion: The encouragement of self-care with prevention minimizes the morbidity and mortality rates related to infections in patients with LPP, reduces health expenses and improves the quality of life of patients with LPP.
\end{abstract}

Keywords: Pressure injury; Nursing; Health education. 


\section{Resumen}

Introducción: la prevalencia de casos de lesiones por presión es exorbitante, y la prevención de las LPP sigue siendo un gran desafío para el enfermero, y su incidencia se considera un indicador de mala calidad de la atención. Objetivos: sin embargo, el objetivo del estudio fue preparar un folleto educativo que enfatice las medidas preventivas para las lesiones por presión en pacientes encamados. Metodología: Se trata de una investigación bibliográfica cualitativa en la que se propuso un folleto educativo para pacientes encamados. Sin embargo, el enfoque metodológico utilizado abordará la investigación bibliográfica como referencia para realizar la misma. Para lograr los objetivos propuestos, la búsqueda se realizó en la base de datos, Google Academic; BVS (Biblioteca Virtual en Salud), SCIELO (Biblioteca Electrónica Científica en Línea), PubMed; LILACS (Literatura Latinoamericana y del Caribe en Ciencias de la Salud). Resultados y discusión: El material presentado y desarrollado para la educación del paciente fue designado a través de un folleto educativo. El material de educación para la salud debe estar redactado de forma sencilla, con un menor nivel de lectura y que permita trasmitir información veraz. Las ilustraciones deben ser atractivas y comunicar claramente su propósito. Además, las imágenes deben alcanzar un alto nivel de atención e interés en la lectura del material, con cambios en la población en los diferentes niveles educativos. Conclusión: El fomento del autocuidado con prevención minimiza las tasas de morbilidad y mortalidad relacionadas con infecciones en pacientes con LPP, reduce los gastos de salud y mejora la calidad de vida de los pacientes con LPP.

Palabras clave: Lesión por presión; Enfermería; Educación para la salud.

\section{Introdução}

A prevalência dos casos de lesão por pressão é exorbitante, e "a prevenção das LPPs continua sendo um grande desafio para os enfermeiros, e sua incidência é considerado um indicador de má qualidade da assistência (Ebi; et al, 2019)". Com isso, Freire (2020) Destaca que mesmo com todo o avanço tecnológico e científico das intervenções em saúde, os índices de lesões por pressão ainda são elevados, variando de $23,1 \%$ a 59,5\%, sobretudo em pacientes de unidade de terapia intensiva (UTI)

As lesões por pressão são de origem multicausal, e para Gonçalves et al. (2020), se dão por fatores intrínsecos como edemas, estado nutricional, idade avançada, incontinência urinaria e fecal, perfusão tecidual diminuída e patologias associadas. E por fatores extrínsecos como cisalhamento, pressão, mobilidade prejudicada, fricção e umidade prolongada da pele. Freire (2020) considera que:

A qualidade da assistência de enfermagem nos serviços de saúde é permeada por esforços para estabelecer diretrizes e protocolos que orientem a prática com o objetivo de reduzir a LPP. Diante deste contexto foi instituído, pela Portaria MS/GM nº 529/2013, o Programa Nacional de Segurança do Paciente (PNSP), com intuito de monitorar a incidência, e instituir planejamento institucional para minimizar este agravo visto que o desenvolvimento das práticas preventivas, durante a internação hospitalar, é um importante indicador da qualidade da assistência.

Diante do exposto, faz-se necessário a pratica das recomendações estabelecidas para avaliar o risco de LPP e uma boa avaliação da pele do paciente no momento da admissão. Isso tornará eficaz o reconhecimento e o tratamento imediato. (Alderden et al. 2017).

O Brasil integra a Aliança Mundial para a Segurança do Paciente, proposta pela Organização Mundial da Saúde, cujo principal propósito é instituir medidas que aumentem a segurança e a qualidade dos serviços de saúde (Mendonça, et al. 2018). $\mathrm{O}$ autor ainda relata que a portaria $\mathrm{n}^{\circ} 524$ e a resolução $\mathrm{n}^{\circ} 36$ publicadas no ano de 2013 pelo MS, orientam as ações necessárias para a segurança do paciente nos serviços de saúde, com a finalidade de promover melhoria do cuidado por meio de protocolos, manuais e guias estabelecidos com foco também nas lesões por pressão.

“Com intuito de reduzir esse agravo na Atenção Primaria em Saúde (APS), o enfermeiro como integrante da Estratégia de Saúde Família (ESF) tem papel fundamental no assistir com melhores práticas de cuidado, propiciando às pessoas e seus familiares se tornarem protagonista no processo Saúde-doença com ações de autocuidado” (Soares \& Heidemann, 2018). 
Assim sendo, a proposta da cartilha educativa se como suporte metodológico para instruir a equipe de saúde, os pacientes e familiares/ou cuidadores. Prevenir e intervir nas LPPs de maneira simples, interativa, sucinta e com conteúdo pratico de fácil acesso e compressão ao leitor.

\section{Materiais e Métodos}

Trata-se de uma pesquisa qualitativa, do tipo bibliográfico, onde foi feito a proposta de uma cartilha educativa para pacientes acamados sobre lesão por pressão, no entanto o percurso metodológico utilizado abordará pesquisa bibliográfica como referência a realização da mesma.

De acordo com Marconi e Lakatos (2013), a pesquisa bibliográfica é um levantamento de toda a bibliografia já publicada, em forma de livros, revistas, publicações avulsas impressas e/ou escrita sobredeterminado tema. Esse tipo de pesquisa compreende oito fases distintas: escolha do tema; elaboração do plano de trabalho, identificação, localização, compilação, fichamento, análise, interpretação e por último a redação.

Após o levantamento bibliográfico acerca do tema em questão, uma proposta de cartilha foi elaborada com a finalidade de fornecer orientações do auto cuidado para não desenvolver a LPP, e dar subsídios para que o leito bem como o paciente acamado possa ter mais independência em seu condicionamento de vida.

Segundo Gil (2009), o desenvolvimento de uma pesquisa bibliográfica varia em função de seus objetivos, ou seja, como os demais tipos de pesquisa, esta é norteada pelos seus objetivos procurando dar conta, através da literatura pesquisada e, posteriormente analisada, de resposta que venham a contribuir com a realidade de problemática enfocada.

Para atingir os objetivos propostos foi realizada uma busca no banco de dados SCIELO; PubMed, LILACS (Literatura Latino-Americana e do Caribe em Ciências da Saúde); Google Acadêmico; BVS (Biblioteca Virtual em Saúde) bem como em livros, para complementar a bibliografia. No período de Janeiro a Novembro de 2021, onde os métodos a serem utilizados na analise dos dados serão através de pesquisa qualitativa, pois se darão por meio da interpretação de dados documentos em livros, artigos científicos, base de dados das ciências biológicas a fim de obter informações necessárias para a pesquisa com intuito de selecionar de forma mais didática o material proposto a ser construído, e assim fazer parte da cartilha.

\section{Resultado e Discussão}

O material elaborado e desenvolvido para educação dos leitores está apontado na cartilha abaixo. 
Figura1 - Cartilha para leitores sobre medidas preventivas de lesão por pressão.

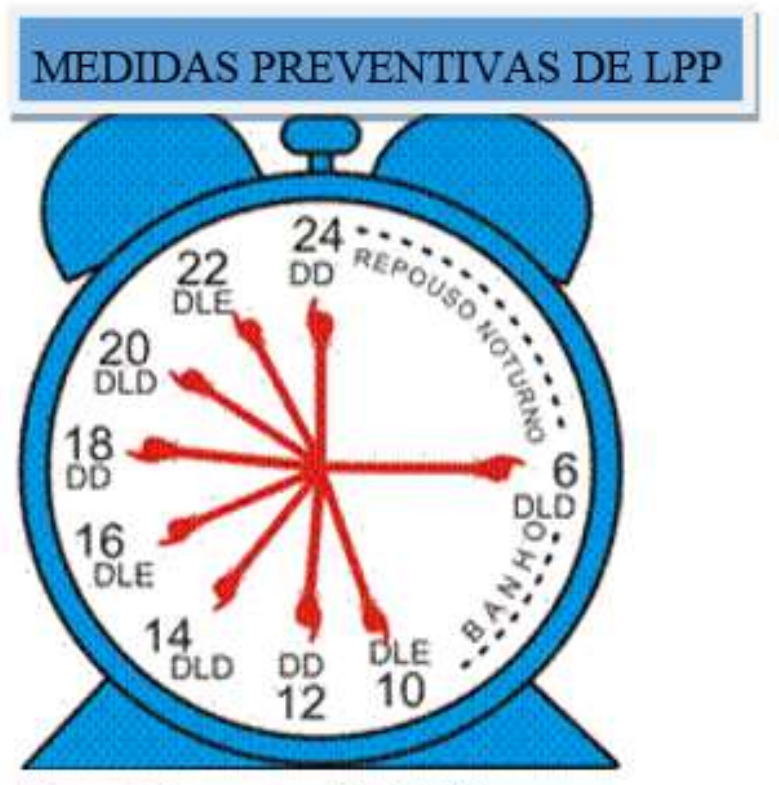

Fonte: Enfermagem Piaui, 2018
Santarém - PA

2021

\section{CENTRO UNIVERSITÁRIO DA AMAZÔNIA - UNAMA CURSO DE BACHARELADO EM ENFERMAGEM}

\section{ANDERSON NASCIMENTO DA CONCEIČÃO \\ ITALO VINICIUS REGIS DOS SANTOS}

Orientadora: Prof. ${ }^{a}$ Enf . Esp. Regiane de Paula Maciel

Santarém - PA

2021 


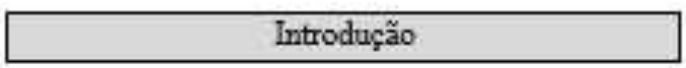

Este material foi desenvolvido, com finalidade extrema de orientar os acompanhantes de pacientes acamados, mostrar as principais causas pelas quais é mais comum o surgimento das LPPs (Lesões por Pressão), pois os mesmos são os mais propicios a desenvolver as LPPs (Lesão por Pressão).

A cartilha trás conteúdo de vinculo educativo, oferecendo ao leito uma visão analitica e autocritica proporcionando asgim um autoconhecimento de como intervir com a prevenção lesão por pressão, juntamente com o profissional enfermeiro.

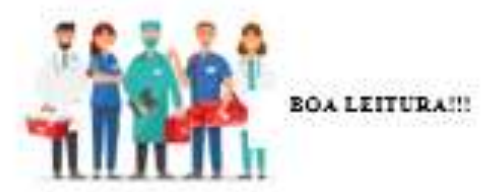

\section{O que é lesão por pressão?}

Lesão por Pressão (LPP) é definida como um dano localizado em tecidos moles elou subjacentes, aberto ou fechado, geralmente sobre uma proeminência óssea, oriundo de pressão prolongada e cisalhamento, o qual pode acarretar isquemia local e morte celular. (Lopes, et, al. 2021).

Diante disso, a LPP configura-se como um evento adverso que contribui significativamente para o risco de infeç̧ão e sepse, podendo prolongar - tempo de internação e os custos dos serviços de saúde (Serra et al., 2018)

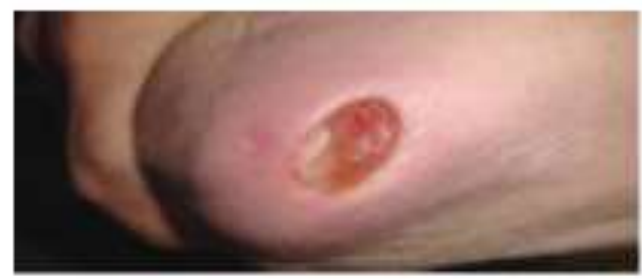

Fonte: Inpuap (2016).

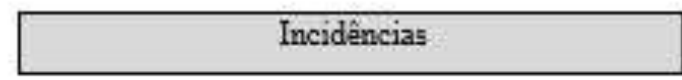

A incidência é maior em individuos acamados, restritos à cadeira de rodas ou que apresentem limitada capacidade de reposicionamento. Recomenda-se a inspeção diária da pele e, principalmente, das áreas que apresentam maior impacto, ou onde são encontradas proeminências ósseas.

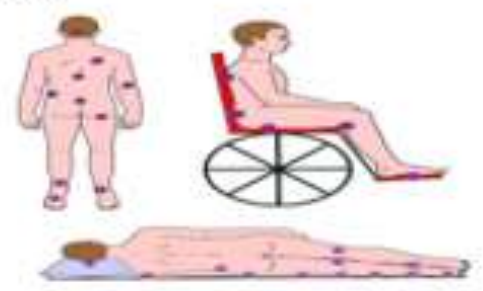

Fonte: Caliri (2018).

- Reposicione o paciente, permitindo o alivio dos pontos de apoio;

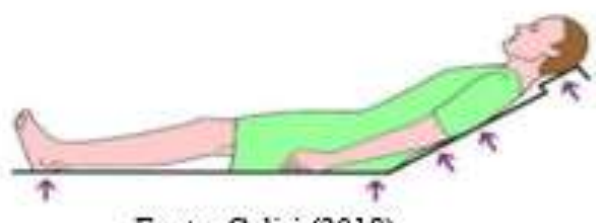

Fonte: Calini (2018).

- A cada 2 horas, mude o paciente de posição;

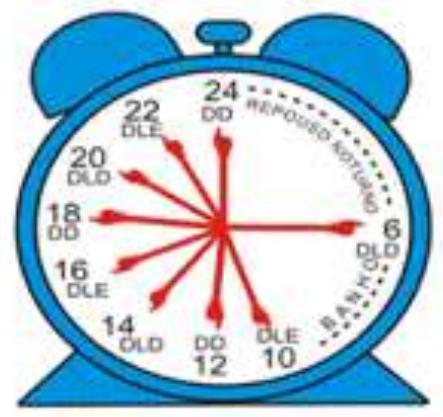

Fonte: Enfermagem Piaui (2018)

- O uso de travesseiros ou coxis é esgencial para manter as proeminências ósgeas livres de pressão e atrito etc.

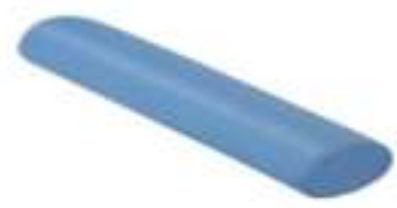

Fonte: Enfermagem Piaui (2018) 


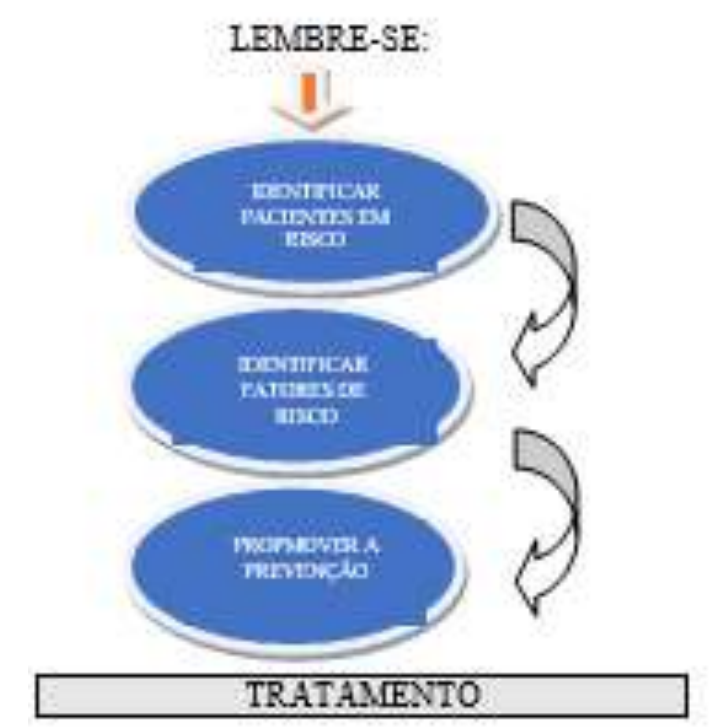

Para o tratamento ideal das LPPs existem quatro principios fundamentais:

1. Patologia de base da LPP deve ser tratada, se possivel.

2. A pressão deve ser alivisda ou removida por medidas adequadas para evitar mais danos teciduais.

3. A nutrição e importante para 2 cicatrização das úlceras por pressão:

- Forrecer calorias suficientes

- Fornecer quantidade adequada de proteinas para o equilibrio positivo de Nitrogênio.

- Forrecer e incentivar a ingestaio diáriz adequada de liquidos para a hidratação

- Fornecer vitaminas e minerais adequados

4. O cuidado da ulcera deve ser otimizado:

- Se houver necrose ou esfacelos, considere o desbridamento para remover o tecido desvitalizado no leito da ferida*

- Limpe a lesão por pressăo e pele ao redor $e$ remova detritos em cada

mudança de curativo para minimizar 2 contaminaçào

- Use coberturas apropriadas para a manutençióo da umidade

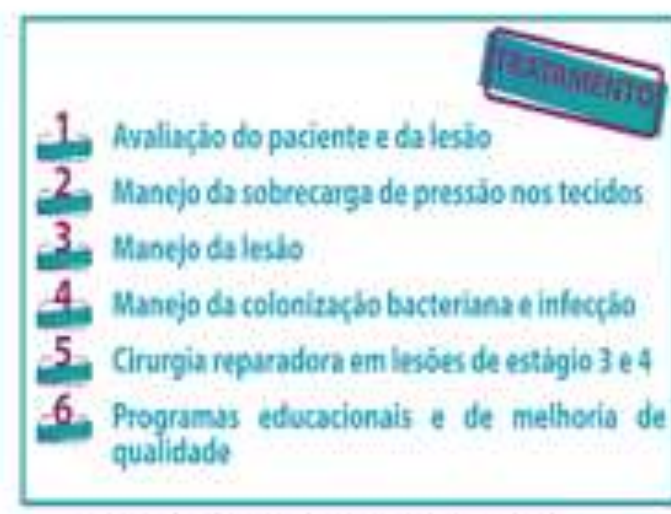

Fonte: Bergatron \& Allman (1992)

\section{REFERENCIAS}

Lopes, H.G. et al Conhecimento dos enfermeiros quanto i identificaçâo e classificaçăo da lezāo por pressão. A enfermagem a partir de uma visão critica: Eșcelência das praticas de cuidado 3, v. 18, n. 1. 11 dez 2018 Acessado em 29/10/2021 as $10: 30$ horas

Serra, R. et al Skin tears and riak factors assesament: a systematic review on evidencebased medicine. Int. Wound J., v. 15, n. 1, P. $38-42,1$ fev. 2018 . DOI: 10.1111 iwj.12815. Acessado em $25 / 11 / 2021$ as $15-50$ horas 
As intervenções de enfermagem para o cuidado com as LPP, requerem acompanhamento da equipe por meio de escalas de medição do risco. Existem na literatura, vários instrumentos para predição desse risco, como a escala de Gosnell, Andersen, Braden, Norton e Walter low (Sanches et al. 2018).

$\mathrm{O}$ autor ainda relata que

“a prevenção da LPP constitui ação primordial, visto que tal agravo causa dor e desconforto para o paciente e família, podendo retardar sua recuperação. O tratamento gera custos à instituição e aumento na demanda de trabalho da enfermagem. Constitui um fenômeno comum a pessoas hospitalizadas em todo o mundo nos diferentes contextos de saúde, especialmente entre os hospitalizados em unidades de terapia intensiva (UTIs), nos quais ela é uma ameaça adicional em doentes já comprometidos fisiologicamente" (Sanches et al. 2018).

De acordo com Mendonça (2018) “os esforços para a elaboração e implantação de protocolos de prevenção de LPP decorrente do Programa Nacional de Segurança do Paciente são notadamente crescentes nos hospitais brasileiros”. O autor ainda relata que a lesão por pressão é um indicador de qualidade da assistência ofertada pelos profissionais responsáveis. Favreto et al. (2017), afirma que os cuidados com as lesões exigem atuação interdisciplinar, adoção de protocolo, conhecimento específico, habilidade técnica, articulação entre os níveis de complexidade de assistência do Sistema Único de Saúde (SUS) e participação ativa dos portadores dessas lesões e seus familiares, dentro de uma perspectiva holística.

O cuidado de enfermagem com as lesões de pele necessita atenção especial por parte dos profissionais da saúde, destacando-se o papel do enfermeiro, que busca novos conhecimentos para fundamentar sua prática. Algumas lesões podem tornar-se crônicas, cuja incidência aumenta gradativamente em todo o mundo, gerando um impacto negativo sobre a qualidade de vida dos pacientes, pois causam dor em diferentes níveis, afetam a mobilidade e possuem caráter repetitivo. Fazendo necessária a sistematização do cuidado com esses pacientes, constituindo, a avaliação da ferida, fator determinante para a terapêutica adequada (Betiolli, et al. 2017).

Cuidados básicos com a pele, como manejo da hidratação também se mostram válidos. A utilização do hidratante é indicada a cada mudança de decúbito, após o banho e sempre que a pele estiver ressecada (Vasconcelos \& Caliri, 2017).

Atenção especial também deve ser dada ao meio ao qual o paciente se encontra manutenção correta das roupas de cama e equipamentos usados para o alívio da pressão em regiões predispostas, bem como a manutenção destes. Os lençóis devem ser dispostos limpos e esticados, visto que pregas e vincos tornam-se zonas de compressão sobre a pele. O leito limpo contribui para o conforto, bem-estar e recuperação do paciente, além de prevenir infecções (Vasconcelos \& Caliri, 2017).

A carência em ações preventivas culmina com o desenvolvimento da LPP e a partir dessa realidade, torna-se necessário à implementação de um tratamento imediato e eficaz que pode minimizar os efeitos nocivos da lesão, tornando mais rápida a recuperação do paciente. (Correia et al, 2019, Manganelli 2019) relatam que o

“... fato de a LPP ser evitável em mais de $95 \%$ dos casos, instigou o interesse em realizar este estudo, uma vez que o enfermeiro é o profissional que está à frente no cuidado ao paciente. Além disso, há escassez de estudos que avaliam a prática do enfermeiro nos cuidados preventivos da LP, o que reforça o interesse desta pesquisa. Ademais, os estudos relacionados às condutas de enfermagem frente à prevenção da LPP destacam a não realização/omissão de cuidados que, em alguns casos, não são percebidos pela equipe de enfermagem”.

De acordo com (Lopes, et al. 2018) a Lesão por Pressão (LPP) é definida como um dano localizado em tecidos moles e/ou subjacentes, aberto ou fechado, geralmente sobre uma proeminência óssea, oriundo de pressão prolongada e cisalhamento, o qual pode acarretar isquemia local e morte celular.

A extensão da perda tecidual é classificada em estágios, sendo do I ao IV. A Lesão por Pressão estágio I é caracterizada como uma lesão com pele íntegra e com eritema não branqueável. O estágio II é uma lesão com perda parcial da 
integridade da pele, ou seja, ocorre a exposição da derme que é a camada intermediária da pele localizada logo abaixo da epiderme e acima da hipoderme, além disso, nesse estágio o leito da ferida apresenta uma coloração rósea ou avermelhada e é úmido, ainda, pode apresentar-se como uma bolha intacta com exsudato seroso ou bolha rompida. (Bordin, et, al. 2017).

No estágio III ocorre a perda total da pele podendo ser visível parte do tecido gorduroso. Já no estágio IV existe a perda total da pele assim como no estágio III, porém, é possível visualizar tendões, cartilagem, ossos e/ou ligamentos (Cabral, et, al. 2021).

Dentre as classificações, existe ainda a Lesão por Pressão não classificável, na qual há perda total da espessura da pele, com presença de esfacelos ou escaras que dificultam a identificação da perda tissular e, a Lesão por Pressão Tissular Profunda que pode apresentar-se em pele íntegra ou rompida, sendo caracterizada por uma região de coloração vermelha escura ou marrom que não embranquece, e que pode apresentar bolha com exsudato sanguinolento (Lima, et,at. 2021).

Os enfermeiros tem a responsabilidade de prever e prover recursos humanos, materiais e estruturais, utilizando dados científicos para implantar medidas preventivas de lesão por pressão. Porém quando o desenvolvimento de lesão por pressão é inevitável, é necessária a adoção de ações terapêuticas adequadas a fim de minimizar as suas consequências e evitar a evolução de sua gravidade. Sendo assim os enfermeiros precisam além do conhecimento técnico científico, possuir informações sobre os custos decorrentes dos cuidados de enfermagem para o tratamento de lesão por pressão a fim de direcionar o uso racional e eficiente de recursos escassos, contribuindo, efetivamente, com o gerenciamento dos custos associados (Campa et, al. 2017).

Diante disso, a LPP configura-se como um evento adverso que contribui significativamente para o risco de infecção e sepse, podendo prolongar o tempo de internação e os custos dos serviços de saúde (Serra et al., 2018). E apesar do caráter preventivo e a constante necessidade de aperfeiçoamento dos cuidados de saúde, a LPP apresenta-se com elevada prevalência, especialmente em idosos hospitalizados (Andrade et al.,2018). Ainda, autores apontam ampla variabilidade de prevalência de LPP em indivíduos hospitalizados, com índices entre 8\% a 40\% (Jaul et al., 2018; Zimmermann et al., 2018).

\section{Considerações Finais}

A prevalência dos casos de lesão por pressão é exorbitante, e a prevenção das LPPs continua sendo um grande desafio para os enfermeiros, e sua incidência é considerado um indicador de má qualidade da assistência.

É de fundamental importância entender a necessidade de serem aplicadas as medidas cabíveis para a prevenção de LPP a qualquer paciente vulnerável, com isso, o trabalho tem como foco principal, elucidar essas medidas preventivas, e levar essas informações as pessoas de uma forma mais simples, através da cartilha educativa.

Entende-se que a LPP pode ser evitada na maioria dos casos, com isso, o trabalho do enfermeiro e da equipe multidisciplinar deve ser realizado com eficiência, para que os números de casos relacionados a LPP venham a atenuar.

\section{Referências}

Alderden, J.et al. (2017). Fatores de risco para lesões por pressão entre pacientes em cuidados intensivos: uma revisão sistemática. Jornal Internacional de Estudos de Enfermagem. 71, 97-114. 10.1016/j.ijurstu.2017.03.012.

Andrade, C. C. D. et al. (2018). Occurrence of pressure ulcer and epidemiological and clinical profile of patients hospitalized in a hospital unit of the Hospitalar de Minas Gerais Foundation. Rev. Méd. Minas Gerais, 28(5), 161-167. 10.5935/2238-3182.20180132.

Bordin, D. et al. (2017). Conhecimento dos enfermeiros quanto à identificação e classificação da lesão por pressão. a enfermagem a partir de uma visão crítica: Excelência das práticas de cuidado 3. RGS.

Betiolli, S. E. et al. (2017). O papel do enfermeiro na prevenção, avaliação e tratamento das lesões por pressão. RGS 17(2):37-47.

Campa, A.et al. (2017). O papel do enfermeiro na prevenção, avaliação e tratamento das lesões por pressão. RGS 17(2):37-47.

Cabral, G. A. et al. Conhecimento dos enfermeiros quanto à identificação e classificação da lesão por pressão. RGS 2021. A enfermagem a partir de uma visão crítica: Excelência das práticas de cuidado 3. 
Correia, A. S. B. et al. (2019). Lesão por pressão: Medidas terapêuticas utilizadas por profissionais de enfermagem. Revista brasileira de ciências da saúde. Paraíba, 23(1). https://doi.org/10.2247/ufpb.2317-6032.2019.

ebi, W. E.; Hirko. G. F.; \& Mijena, D. A. (2019). Conhecimentos de enfermeiros para a prevenção de úlceras por pressão em hospitais públicos de Wollega: desenho de estudo transversal. BMC NURS. 18.

Favreto, F. J. L., et al. (2017) O papel do enfermeiro na prevenção, avaliação e tratamento das lesões por pressão. RGS 17(2):37-47.

Freire, D. A. et al. (2020). Variáveis associadas a prevenção das lesões por pressão: Conhecimento para o cuidado de enfermagem. Revista online de pesquisa cuidado é fundamental. http://dx.doi. Org/09789/2175-5361.

Gil, A. C. (2002). Como elaborar projetos de pesquisa. (4a ed.) Atlas.

Jaul, E. et al. (2018). An overview of co-morbidities and the development of pressure ulcers among older adults. BMC Geriatr., 18(1) 1. 10.1186/s12877-0180997-7.

Lima, M. L., et al. (2021). Conhecimento dos enfermeiros quanto à identificação e classificação da lesão por pressão. A enfermagem a partir de uma visão crítica: Excelência das práticas de cuidado 3(1)

Lopes, H. G. et al. (2018). Conhecimento dos enfermeiros quanto à identificação e classificação da lesão por pressão. A enfermagem a partir de uma visão crítica: Excelência das práticas de cuidado 3. 18(1),11.

Manganelli, R. R. et al. (2018). Intervenções de enfermeiros na prevenção de lesão por pressão em uma unidade de terapia intensiva. Revista de enfermagem da ufsm. Santa maria- RS.

Mendonça, P. K. et al. (2018). Ocorrência e fatores de risco para lesões por pressão em centros de terapia intensiva. Revista de Enfermagem. Recife-PE. https://doi.org/10.5205/19818963-v12i2a23251p303-2018.

MEndonça, P. N. (2018). Prevenção de lesão por pressão: Ações prescritas por enfermeiros de centros de terapia intensiva. Texto e Contexto-Enfermagem. 27(4). Florianópolis, https://doi.org/10.1290/0104-07072018004610017

Marconi, M. A.; \& Lakatos, E. M. (2003). Fundamentos de Metodologia cientifica. (6a ed.) Editora Atlas.

Mendonça, P. K., Loureiro, M. D. R., Ferreira Júnior, M. A., et al. Ocorrência e fatores de risco para lesões por pressão em centros de terapia intensiva. Revista de Enfermagem da UFPE, 12(2): 303-311.

Sanches, O. B., et al. Adesão da enfermagem ao protocolo de lesão por pressão em unidade de terapia intensiva. Archives of Health Sciencis-AHS. São José do rio preto-SP. 2018. doi.org/10.17646/2318-3691.25.3.2018.1058.

Serra, R. et al. Skin tears and risk factors assessment: a systematic review on evidence-based medicine. Int. Wound J., 15(1),38-42, 10.1111/iwj.12815.

Soares, C. F.; \& Heidemann, I. T. S. B. (2018). Promoção da saúde e prevenção da lesão por pressão: expectativas do enfermeiro da atenção primária. Universidade Federal de Santa Catarina. Florianópolis-SC. http://dx.doi.org/10.1590/0104070720180001630016.

Vasconcelos, J. M. B. et al. (2017). Ações de enfermagem antes e após um protocolo de prevenção de lesões por pressão em terapia intensiva. Escola Anna Nery.

VasconceloS J. M. B., \& Caliri, M. H. L. (2017). Ações de enfermagem antes e após um protocolo de prevenção de lesões por pressão em terapia intensiva. Revista Escola Anna Nery de Enfermagem, 21(1): 1-9.

Zimmermann, G. S. et al. (2018). Pressure injury risk prediction in critical care patients: Anintegrative review. Texto e Contexto Enf., 27(3), e3250017, $10.1590 / 0104-07072018003250017$ 